Documentation et bibliothèques

DOCUMENTATION BIBLIOTHËQUES

\title{
La référence automatisée dans les bibliothèques d'université et de recherche
}

\section{Claude Bonnelly}

Volume 19, numéro 1, mars 1973

URI : https://id.erudit.org/iderudit/1050960ar

DOI : https://doi.org/10.7202/1050960ar

Aller au sommaire du numéro

Éditeur(s)

Association pour l'avancement des sciences et des techniques de la documentation (ASTED)

ISSN

0315-2340 (imprimé)

2291-8949 (numérique)

Découvrir la revue

Citer cet article

Bonnelly, C. (1973). La référence automatisée dans les bibliothèques d'université et de recherche. Documentation et bibliothèques, 19(1), 26-33. https://doi.org/10.7202/1050960ar
Résumé de l'article

Etude de l'automatisation comme solution aux problèmes que rencontrent aujourd'hui les services de référence dans les bibliothèques d'université et de recherche. Analyse de ses diverses possibilités : automatisation du service comme tel, utilisation de banques de données spécialisées à des fins de référence, exploitation des services de diffusion sélective de l'information (SDI). Ce que doit connaître le bibliothécaire pour exploiter à fond ces systèmes : caractéristiques des diverses espèces d'index, techniques d'élaboration d'un profil d'intérêt.
Tous droits réservés (C) Association pour l'avancement des sciences et des techniques de la documentation (ASTED), 1973
Ce document est protégé par la loi sur le droit d'auteur. L'utilisation des services d'Érudit (y compris la reproduction) est assujettie à sa politique d'utilisation que vous pouvez consulter en ligne.

https://apropos.erudit.org/fr/usagers/politique-dutilisation/ 


\section{La référence automatisée dans les bibliothèques d'université et de recherche}

\author{
par Claude Bonnelly \\ Ecole de bibliothéconomie \\ Université de Montréal
}

Etude de l'automatisation comme solution aux problèmes que rencontrent aujourd'hui les services de référence dans les bibliothèques d'université et de recherche. Analyse de ses diverses possibilités: automatisation du service comme tel, utilisation de banques de données spécialisées à des fins de référence, exploitation des services de diffusion sélective de l'information (SDI). Ce que doit connaître le bibliothécaire pour exploiter à fond ces systèmes: caractéristiques des diverses espèces d'index, techniques d'élaboration d'un profil d'intérêt.

Norman Hoyle, dans l'article "Academic Library Reference Service", décrit ainsi la bibliothèque d'université et son service de référence:

"An academic library is several libraries at once. It is a public library in that it serves the campus community as a general intellectual and cultural facility. It is a school library in that it is geared toward an instructional program, providing students with assistance in completing course assignments. It is a special library in that it meets the very specialized information and resource needs of graduate students and faculty who are engaged in advanced research. The great problem faced by the reference department in an academic library is not so much that its tasks are unique, but that its tasks are so many, and that its clientele are so diverse."1

Les services de référence dans les bibliothèques universitaires font donc face à de nombreux problèmes et doivent continuellement se redéfinir pour pouvoir rejoindre convenablement leur clientèle.

En 1955, Samuel Rothstein publiait une étude exhaustive sur les services de référence dans les bibliothèques américaines. ${ }^{2}$ II y notait les grandes lignes d'évolution qu'ils ont connu depuis leur origine. Cette évolution peut se résumer en deux points:

1) passage graduel d'une référence indirecte à une référence directe;

1 Norman HOYLE "Academic Library Reference Service", In William A. Katz, Introduction to Reference Work, N.Y., MCGraw-Hill, 1969, II:145.

2. Samuel ROTHSTEIN, The Development of Reference Services Through Acedemic Traditions, Public Library Practice and Special Librarianship, Chicago, ACRL, 1955, 124p.
2) passage d'une référence générale à une référence de plus en plus spécialisée.

Au début de leur existence, les services de référence ne donnaient en général qu'un service d'orientation au lecteur, c'est-à-dire qu'ils ne faisaient que le guider dans l'utilisation de la bibliothèque et de la documentation, afin qu'il se débrouille par lui-même. Ce genre de service existe toujours certes, mais avec le temps, la référence a dû s'orienter aussi vers un service de plus en plus direct, consistant non seulement à guider le lecteur, mais de plus à maintes occasions, à lui fournir directement les informations qu'il cherchait.

En même temps, la référence se devait de devenir de plus en plus spécialisée. Au début, le bibliothécaire de référence pouvait n'avoir que de bonnes connaissances générales, et cela n'avait pas trop d'inconvénients. Mais, pour suivre l'évolution de l'enseignement et de la recherche et pouvoir donner des informations directes et adéquates au lecteur, il se devait d'être au même niveau que lui, afin de bien comprendre ses questions; il devait donc posséder une connaissance approfondie des diverses disciplines d'enseignement et de recherche.

Ces changements étaient dus aux besoins sans cesse grandissants et de plus en plus complexes des professeurs et des étudiants, surtout au niveau des études supérieures et de la recherche. En conséquence, rares sont aujourd'hui les services de référence dans les bibliothèques universitaires qui ne possèdent pas quelques bibliothécaires ayant une formation spécialisée dans l'une ou l'autre des disciplines.

En 1973, les lignes d'évolution sont toujours les mêmes; bien plus, elles se sont accentuées à un point tel que de nouvelles orientations doivent se dessiner pour y faire face. En effet, les chercheurs assistent aujourd'hui à une explosion continuellement croissante de la documentation; non seulement ils s'en remettent de plus en plus à la bibliothèque dans l'élaboration de leur recherche, mais ils exigent un service plus rapide et plus efficace, et ce, surtout dans le domaine des sciences et des techniques où les connaissances et la documentation deviennent vite désuètes.

"The past quarter century and, more particularly, the past few years, have witnessed profound changes in the role of libraries in institutions of higher education...

This change... can largely be attributed to the fact that the rising tide of publications and the resulting growth of library collections created numerous pressures on the traditional library economy. The result of these pressures was felt by research and technical libraries... in their increasing difficulty to respond to individual requirements in a speedy and effective manner." 3

3. Burton W. ADKINSON, and Henry J. DUBESTER, "The New Challenges for University and Technical Libraries". Libri, vol. 19 (1969), 265. 
La solution que l'on apporte généralement pour répondre à ces besoins croissants de rapidité et d'efficacité est l'automatisation de la référence. Les bibliothécaires en général la reconnaissent et mettent en elle beaucoup d'espoir. C'est là une conclusion que rapportait Marguerite Densky dans la compilation des résultats d'une enquête effectuée en 1968 sur les services de référence dans les bibliothèques canadiennes:

"Tous les bibliothécaires consultés se disent en général satisfaits des services qu'ils offrent présentement et qui semblent répondre aux exigences de leur public. Toutefois, tous attendent beaucoup de l'avenir et basent leurs espoirs surtout sur l'ordinateur. Celui-ci devrait libérer le bibliothécaire de toutes les tâches routinières et l'aider à accumuler et à organiser les informations." 4

Mais qu'est-ce que la référence automatisée? Quelles sont ses caractéristiques propres? Dans une première partie, nous étudierons les diverses possibilités d'automatisation de la référence et les réalisations déjà faites en ce domaine. Dans une seconde partie, nous essaierons d'analyser, dans ses grandes lignes, le travail et les connaissances que doit fournir le bibliothécaire pour pouvoir exploiter à fond les systèmes de référence automatisée.

\section{LA REFERENCE AUTOMATISEE: SES CARACTERISTIQUES ET POSSIBILITES.}

\section{1) Automatisation globale de la référence}

On parle beaucoup de la référence automatisée, de ses avantages, de ses possibilités. Toutefois, l'automatisation complète et totale d'un service de référence n'est pas pour demain. Poussée à l'extrême en effet, elle signifierait l'avènement de la bibliothèque d'ordinateur, sans livres et sans bibliothécaires. Toutes les connaissances existantes seraient emmagasinées dans une mémoire d'ordinateur, et le chercheur n'aurait qu'à formuler sa question sur un clavier pour aussitôt recevoir une réponse adéquate et exhaustive. On voit tout de suite la complexité de la réalisation d'un tel système. Des expériences toutefois sont tentées en ce sens. Ainsi en est-il des travaux en cours au Massachussets Institute of Technology, d'où est sorti le projet INTREX:

"The Massachussets Institute of Technology, for example, is experimenting with a program called information transfer or INTREX. The problem MIT has set itself is to devise a system whereby a user might carry on a dialogue with a computer, just

\footnotetext{
4. Marguerite DENSKY, "Les services de référence dans les bibliotheques canadiennes", Bulletin de l'ACBLF, vol. 16. no 2 (juin 1970), 75 .
}

as he now talks to a reference librarian. For example, the user might employ a ty. pewriter keyboard to phrase his questions. The typewriter would be on a line with a computer which would consider the question, search its memory (reference sources) and give a reply in the form of a printed-out statement, or possibly on a television screen. The ultimate hope is that such a system would be able to cope with this example: "What important new developments have there been in the theory of social interactions?" the patron would ask the machine. And the machine would then give a clear, carefully constructed 200 word essay discussing the new ideas that have been developed." 5

L'immense variété des questions auxquelles doivent répondre les services de référence des bibliothèques universitaires rend la réalisation d'un tel système quasi impossible, du moins dans l'immédiat.

\section{2) Automatisation graduelle de la référence}

Certains auteurs préconisent une approche plus réaliste du problème. Une méthode proposée consisterait à emmagasiner dans la mémoire de l'ordinateur les réponses aux questions de référence, à mesure qu'elles sont données à l'usager. Au bout d'un certain temps, l'ordinateur contiendrait un certain bagage d'information qui continuerait à s'accroître indéfiniment. C'est ce que proposent, entre autres, Heiliger et Henderson:

"The problem of repeat questions is now handled in some libraries with a special card file. The reference librarian on duty writes the question on a card and indicates where the answer was found. The next librarian to come on duty scans the new cards and files them away. There is the possibility, with on-line access to the computer, of keeping such a file in computer store. Before tackling a new question, the reference librarian could search the record for any previous similar questions and readout or printout the results of the past effort." 6

Mais une telle solution pose aussi de graves problèmes, étant donné la grande variété des questions posées. Comment entrer les réponses dans l'ordinateur, comment les indexer? Comment les caractériser? Et surtout, comment les mettre à jour? La tâche est énorme et nécessite l'élaboration de programmes complexes.

5. William A. KATZ, Introduction to.., II; 216

6. Edward M. HEILIGER and Paul B. HENDERSON Jr., Library Automation: Experience, Methodology, and Technology of the Library as an Information System, New-York, McGraw-Hill, 1971, p.55. 
3) Exploitation de banques de données spécifiques à des fins de
référence

L'automatisation globale ou graduelle de la référence comme telle reste donc, dans l'immédiat, un problème assez complexe; elle est d'ailleurs critiquée par certains auteurs, tel Prodrick qui affirme que:

"The input aspect of this would seem excessively complex, and the output aspect complex, rigid, time-consuming and expensive and so, as a basis for general reference service, quite impossible."7

Plusieurs auteurs préconisent pour l'immédiat une approche différente qui consiste, non pas à automatiser les services de référence comme tels, mais plutôt à utiliser et à exploiter des banques de données automatisées dans divers secteurs, à des fins de référence. C'est d'ailleurs en ce domaine qu'il y a le plus de réalisations concrètes. Insistons un peu sur ce point.

L'usager fait appel à un service de référence pour obtenir soit des données d'information, soit des données bibliographiques. Voyons quels sont les systèmes d'automatisation possibles dans ces deux catégories de recherche.

\section{A) Recherche d'informations}

Prodrick mentionne la possibilité de constituer sur ordinateur des banques d'information compilant diverses catégories spécifiques de données, comme par exemple des statistiques, des tables mathématiques, des formules chimiques, des données biographiques, des faits et dates historiques, des cas de jurisprudence, etc... ${ }^{8}$ Ici au Québec, le projet DATUM (Documentation Automatique de Textes juridiques de l'Université de Montréal) constitue un bon exemple d'une banque de données dans le domaine de la jurisprudence, banque qui pourrait avantageusement être exploitée dans les bibliothèques de droit. Ainsi, les bibliothèques possédant un ordinateur pourraient établir de telles banques spécifiques, ou encore acheter et exploiter des banques produites par d'autres organismes.

\section{B) Recherche documentaire}

Les réalisations sont encore plus nombreuses dans le domaine de la recherche documentaire, c'est-à-dire la compilation de données bibliographiques.

Par exemple, une bibliothèque qui a déjà automatisé son service de catalogage et de classification peut facilement, si elle le désire, utiliser la mémoire ainsi constituée pour en tirer des bibliographies à l'intention de ses usagers, et ce, de façon très rapide. Si, en outre, cette bibliothèque fait partie d'un réseau relié directement à un ordinateur central, il devient possible alors de compiler rapide-

\footnotetext{
7. R.G. PRODRICK, "Automation Can Transform Reference Services", Ontario Library Review (Sept. 1967), 150.

8. Ibid., 150.
}

ment des bibliographies spécifiques des ressources disponibles dans les diverses bibliothèques faisant partie du réseau, ce qui à une répercussion immédiate sur le prêt entre bibliothèques.

Une autre application possible est celle de la signalisation courante de la documentation ou SDI (Selective Dissemination of Information). Les chercheurs qui désirent se mettre continuellement à jour dans leur domaine de recherche consultent en général régulièrement divers index, bulletins bibliographiques ou abstracts. Or il arrive que, de plus en plus, pour faire face à l'explosion croissante de la documentation, ces abstracts soient compilés et produits par ordinateur. C'est le cas, par exemple, de Chemical Abstracts, Physics Abstracts, Engineering Index, Index Medicus, Biological Abstracts, et de bien d'autres $^{9}$ Souvent, les producteurs de ces sources bibliographiques offrent aussi à leurs usagers un abonnement à ces bandes magnétiques. Les bibliothèques pourraient donc les acheter, les exploiter sur ordinateur et offrir ainsi à leur clientèle un service plus rapide et plus efficace.

Evidemment, toutes les bibliothèques ne peuvent pas se permettre d'offrir un tel service. Primo, l'investissement initial est assez élevé. Secundo, il y a actuellement un manque total de normalisation des bandes produites par les divers organismes, de sorte que, souvent, il faut prévoir l'établissement de programmes distincts pour traiter les diverses bandes, ce qui augmente la complexité du service, et les coûts.

"The first and foremost problem derives from the fact that libraries were in no position to cope with this type of information resource. It is one thing to have a computer which is used for the internal operations of the library, even including certain of the innovative bibliographic operations such as are involved with the MARC program. It is quite another to develop a capability which entails acquisition of tapes from a variety of sources, involving variations in initial computer hardware, in format of the digitalized contents, and differences in the programs needed to process the tapes." 10

Plusieurs solutions peuvent être envisagées pour résoudre ce problème. L'une est extérieure aux bibliothèques et concerne la normalisation des divers services documentaires existant.

"Producers of information services are beginning to realize that their tape products will be used at a common point and that standardization of format and of other aspects of their products will become more and more imperative."11

En 1961, I'UNESCO, en collaboration avec le CIUS (Conseil International des

9. Burton W. ADKINSON, et Henry J. DUBESTER, "The New Challenges for ... "269.

10. Ibid., 269.

11. Ibid., 269. 
Unions Scientifiques) faisait une étude sur la réalisation d'un système mondial d'information scientifique. L'une des recommandations de cette étude concerne justement la normalisation des services documentaires. II s'agit de la recommandation numéro 2:

"Des consultations d'experts devraient être maintenues d'une façon permanente dans le cadre de l'Unisist pour accélérer les efforts internationaux, par l'intermédiaire de l'Organisation Internationale de Normalisation (ISO), visant à la normalisation des éléments bibliographiques pour le transfert international de l'information, à l'adoption de formats et de codes normalisés pour la représentation de ces éléments dans les systèmes mécanisées, à l'unification des règles de translittération et des caractères typographiques et autres sujets connexes." 12

C'est là un premier pas à réaliser.

Les autres solutions concernent directement les bibliothèques. L'une, proposée par Adkinson et Dubester, consisterait à étendre les services offerts à une clientèle extérieure à l'université (industries, centres de recherche, associations professionnelles, etc...), en exigeant des frais de service; une telle ouverture augmenterait la rentabilité du système.

Une autre solution consisterait à créer un réseau de bibliothèques participant à un programme commun d'exploitation de la documentation automatisée. Au Canada par exemple, la Bibliothèque scientifique nationale offre depuis plusieurs années aux chercheurs canadiens un service de signalisation courante de la documentation par ordinateur, le service CAN/SDI. Ce service reçoit et exploite à l'intention de ses usagers les bandes provenant de divers organismes. Ainsi, il reçoit actuellement les bandes produites par I'Institute of Scientific Information (Philadelphie), soit les bandes magnétiques correspondant aux ISI Current Contents, ISI Science Citations Index, ISI Permuterm Index, ISI Source Index. II traite egalement les bandes correspondant aux publications suivantes: Bibliography and Index of Geology, Biological Abstracts et Bioresearch Index, Chemical Abstracts, Chemical Titles, Engineering Index (Service COMPENDEX), Index Medicus (Service MEDLARS), Science Abstracts: Section A: Physics, Section B: Electrical and Electronics, Section C: Computer and Control (Service ISPEC). II exploite également les bandes MARC II provenant de la bibliothèque du Congrès (Washington), de même que les bandes provenant du PIP (Pollution Information Project, NRC, Ottawa). Ces diverses bandes étant offertes sous des formats différents, les responsables de CAN/SDI ont élaboré un programme spécial pour les convertir en un format unique, le format MARC. Le traitement

12. UNISIST; abrégé de l'étude sur la réalisation d'un système mondial d'information scientifique, effectuée par l'Organisation des Nations Unies pour I'Education, la Science et la Culture et le Conseil International des Unions Scientifiques, Paris, Unesco, 1971, p.45. en est ainsi avantageusement simplifié. ${ }^{13}$

II serait alors souhaitable que les bibliothèques de recherche du pays qui désireront offrir un tel service, profitent du travail déjà fait et collaborent avec la Bibliothèque scientifique nationale dans l'exploitation de ces systèmes. C'est ce qu'a fait, par exemple, la bibliothèque de l'Université Laval en créant le groupe GESYDAMS (Groupe d'Exploitation des SYstèmes de Documentation Automatisés en Médecine et en Sciences). Ce groupe comprend les bibliothécaires de référence en sciences et en médecine; ces bibliothécaires servant d'intermédiaires entre les chercheurs de l'Université et le service CAN/SDI: ils informent l'usager, analysent ses demandes, construisent les profils d'intèrêt, transmettent les profils à CAN/SDI, évaluent la rentabilité du profil d'intérêt, etc...

\section{CONNAISSANCES REQUISES DU BIBLIOTHECAIRE DANS L'EXPLOITATION DES SYSTEMES DE DOCUMENTA - TION AUTOMATISEE}

La référence automatisée, loin d'être une simple projection pour l'avenir, est donc en partie réalisée, surtout en ce qui concerne la recherche documentaire ou bibliographique. Nous avons vu en effet que plusieurs services documentaires sont maintenant automatisés, et offrent la possibilité d'une recherche bibliographique par ordinateur, plutôt que par compilation manuelle des index et répertoires. Certains services n'offrent qu'une signalisation courante; d'autres offrent aussi la possibilité d'une recherche documentaire rétrospective: c'est le cas notamment du service MEDLARS, qui publie I'Index Medicus.

Toutefois, afin de pouvoir exploiter à fond ces systèmes de documentation automatisée au profit de sa clientèle, le bibliothécaire de référence doit posséder certaines connaissances de base indispensables. Bien sûr, il doit d'abord connaître quels sont les index ou abstracts qui sont, de fait, automatisés tout comme, dans la référence traditionnelle, il devait connaître de son mieux les titres des divers répertoires et index dans différents domaines. II doit donc faire un effort de recherche de ce côté. Mais en plus, s'il veut servir l'usager avec un maximum d'efficacité, il lui faut maîtriser les connaissances suivantes:

1) il doit connaître les diverses espèces d'index produits par ordinateurs: leurs avantages, leurs inconvénients, leur force, leurs limites respectives, et ceci afin de pouvoir exploiter à fond les systèmes qu'il aura choisis;

2) il doit savoir transmettre de la meilleure façon possible la question de l'usager à la mémoire de l'ordinateur: il doit donc savoir ce qu'est un profil d'intérêt et en connaître les règles d'élaboration.

13. J. HEILIK, "Information Retrieval and MARC at the National Science Library", Canadian Library Journal (1971), 120-123. 


\section{1) Connaissance des diverses espèces d'index ${ }^{14}$}

On peut d'abord regrouper les index existant (bulletins bibliographiques, abstracts, etc...) en deux grandes catégories: ceux qui utilisent un vocabulaire libre, et ceux qui utilisent un vocabulaire contrôlé. Les index à vocabulaire libre sont basés sur le langage naturel: la recherche documentaire s'y fait à partir des mots ou des concepts employés couramment par les lecteurs et les auteurs des documents. L'index à vocabulaire contrôlé est plus systématisé: il repose sur un vocabulaire préalablement défini et accepté, plus ou moins fermé, plus ou moins structuré, que l'on présente habituellement sous forme de thesaurus. Avant d'entreprendre une recherche documentaire, il faut, dans ce cas, traduire le langage naturel employé par l'usager dans le langage documentaire défini, et ce à l'aide du thesaurus des mots-clef.

Ces deux grandes catégories d'index ont des avantages et des inconvénients respectifs. L'index à vocabulaire libre a l'avantage d'être très près de l'usager, qui aura ainsi accès à la documentation sans avoir à comparer les termes qu'il emploie avec ceux d'un thesaurus parfois fort complexe. Par contre, il a l'inconvénient de reposer sur le langage naturel qui est parfois assez ambigu. Un concept en effet peut s'exprimer dans le langage naturel par plusieurs mots plus ou moins synonymes:

"par exemple, une réunion de personnes qui se rassemblent pour échanger leurs idées ou se communiquer leurs études est, suivant la fantaisie des organisateurs, un congrès, un colloque, une conférence, un symposium, un séminaire, un débat, une réunion ou un groupe de travail." 15

Inversement, un mot du langage naturel peut exprimer parfois plusieurs concepts très différents; ainsi

"un séminaire sera, suivant le contexte, un établissement d'enseignement religieux ou un groupe de travail pour l'étude de certaines questions." 16

Dans ces cas, l'index à vocabulaire contrôlé n'emploiera qu'un seul terme accepté, auquel tous les autres seront renvoyés. Ces distinctions sont importantes à connaître car, si l'on effectue une recherche à l'aide d'un index à vocabulaire libre, il faudra au préalable analyser le concept que l'on a en tête, pour en rechercher toutes les expressions possibles dans le langage naturel.

14. Les sources qui ont servi à l'élaboration de cette partie sont les suivantes: Jacques CHAUMIER, Les techniques documentaires, Paris, Presses Universitaires de France, $1971,128 p$.

F. Wilfrid LANCASTER, Information Retrieval Systems: Characteristics, Testing and Evaluation, New-York, John Wiley, 1968, 222p.

mais surtout: Marcel VAN DIJK, et Georges VAN SLYPE Le service de documentation face à l'explosion de l'information, Paris, Editions d'Organisation, 1969, 265 p. $2 e$ partie, ch. 4: "Choix du langage d'indexation", p.47-68.
L'index à vocabulaire contrôlé, pour sa part, a l'inconvénient d'être fermé. II sera donc très efficace dans le cas d'une discipline où le langage est déjà très structuré et d'une évolution lente. II le sera par contre moins dans une discipline où le langage n'est pas clairement défini, et où il évolue si rapidement que le thesaurus des mots-clef est toujours, ou presque toujours, dépassé.

Après avoir vu ces caractéristiques générales, voyons maintenant quelles sont les différentes espèces d'index dans chacune de ces deux catégories.

\section{A) Vocabulaire contrôlé}

Les index à vocabulaire contrôlé peuvent se présenter sous forme d'index à classification contraignante ou à classification noncontraignante. Les index à classification contraignante reposent sur un système de classification hiérarchique comme par exemple les systèmes Dewey, LC et CDU, où tous les concepts sont regroupés à l'intérieur de classes et de sous-classes. Les index à classification non-contraignante reposent sur une liste contrôlée de mots ou de descripteurs-clef, présentés dans un ordre alphabétique: il n'est pas question dans ces index de placer le concept à l'intérieur d'une classe. L'Index Medicus et son thesaurus en constituent un bon exemple.

Les index à classification non-contraignante sont beaucoup moins rigides que ceux à classification contraignante. Toutefois, les index à classification hiérarchique offrent souvent la possibilité d'une recherche à partir de vedettes-matière ou descripteurs-clef.

\section{B) Vocabulaire libre}

Les index à vocabulaire libre se présentent sous forme d'index à descripteurs libres ou encore sous forme d'index KWIC (Key-word in context). Dans les premiers, le contenu des documents est analysé et indexé, mais à l'aide des termes ou descripteurs employés par l'auteur lui-même. L'index des sujets du Chemical Abstracts est, à quelques variations près, fondé sur ce principe.

Dans les index conçus sur le modèle $K W I C$, les documents ne sont pas analysés et indexés par une personne intermédiaire. En effet, ce sont les mots mêmes du titre et parfois ceux du résumé, qui servent de termes d'indexation. Dans ce cas, le bulletin imprimé présente une suite de titres ou de références bibliographiques dont l'un des termes apparaît toujours en caractères gras, ou un peu en retrait.

"Le titre apparaît dans l'index autant de fois qu'il comporte de mots significatifs, ces derniers étant placés toujours au même endroit en colonne centrale. Ainsi pour un article ayant pour titre: "Utilisation de l'algèbre de Boole en documentation automatique sur ordinateur", il y aurait....

15. Marcel VAN DIJK, et Georges VAN SLYPE, Le service de documentation ..., 49-50.

16. Ibid., 50 . 
Itilisation de I'ALGEBRE de Boole en dor xcumentation AUTOMATIQUE sur ordinat 'algèbre de BOOLE en documentation : e Boole en DOCUMENTATION automa omatique sur ORDINATEUR

...Chaque mot fait office de descripteur et a son sens explicité par le contexte qui l'entoure."17

L'ordinateur fera la recherche documentaire, non pas en lisant les descripteurs emmagasinés dans sa mémoire et préalablement extraits des documents, mais en lisant successivement tous les titres pour s'arrêter à ceux qui contiennent les termes qu'on lui aura dictés.

Les index KWIC ont l'avantage de pouvoir être produits très facilement et très rapidement par l'ordinateur lui-même, sans qu'on ait besoins d'indexeurs pour analyser chacun des documents et en tirer des descripteurs. Cet avantage répond aux demandes croissantes de rapidité venant des usagers. Ils ont toutefois un grand inconvénient: la valeur de ces index dépend de la qualité et de la pertinence des titres que les auteurs ont donné à leurs documents. Plusieurs études statistiques ont été entreprises, en vue de mesurer justement si les termes utilisés dans les titres correspondaient en général assez bien à la teneur des articles. Dans Information Retrieval Systems, p.114, Lancaster donne une bibliographie de quelques-unes de ces études. Les opinions émises sur ce point sont assez partagées. Mais, comme le note Chaumier, "le cas le plus fréquent d'utilisation concerne l'index des titres d'articles de périodiques"; or, les "titres d'articles [sont] en principe plus explicites et plus précis que pour d'autres documents." 18 "I semble donc que, en général, les avantages compensent de beaucoup les inconvénients.

De toute façon, une grande quantité d'index, et parmi les plus importants, sont maintenant préparés par ordinateur selon la méthode KWIC. Qu'il suffise par exemple de mentionner Chemical Titles et surtout l'index des sujets de Biological Abstracts.

II existe enfin une troisième catégorie d'index, un peu spéciale: l'index des citations. Cette forme d'index sert à repérer tous les articles où l'on cite un document donné. Elle correspond à une méthode assez courante chez les chercheurs, méthode qui consiste à repérer la documentation pertinente en se fiant aux citations faites dans les documents. Elle repose sur l'hypothèse suivante: si un auteur cite une étude considérée comme très importante dans l'étude de tel secteur, il y a de fortes chances pour que le texte de cet auteur couvre et fasse avancer le même domaine de recherche. Science Citation Index est le meilleur exemple de cette forme d'index.

17. Jacques CHAUMIER, Les techniques..., 97-98.

18. Ibid., 96.
Les services documentaires offrant un repérage de la documentation par ordinateur optent pour l'une ou l'autre de ces formules dans l'enregistrement de leurs données.

\section{2) Connaissances relatives à l'élaboration d'un profil d'intérêt}

Après avoir maîtrisé la connaissance des diverses formes d'index, le bibliothécaire de référence pourra beaucoup plus facilement connaître les limites et possibilités des divers systèmes qu'il sera appelé à utiliser; il pourra même prévoir un peu leur rentabilité respective. Mais pour les exploiter à fond, il aura besoin d'autres connaissances encore plus importantes. Ces connaissances ont trait à la manière de transmettre la question de l'usager à la mémoire de l'ordinateur, pour en obtenir les réponses les plus pertinentes, de la manière la plus efficace. Ce sont donc des connaissances relatives à l'élaboration des profils d'intérêt.

Jacques Chaumier, dans son ouvrage Les techniques documentaires, traite un peu de cette question, de même que Lancaster dans Information Retrieval Systems; mais la source la plus importante est un manuel publié par le service CAN/SDI de la Bibliothèque scientifique nationale et qui s'intitule Profile Design Manual (une 3e édition est parue en 1972). Pour concrétiser les règles générales d'élaboration d'un profil, nous partirons d'un exemple. Supposons un lecteur qui désire qu'on lui signale des articles courants sur "l'audio-visuel comme méthode d'enseignement dans les universités et les collèges". Comment transmettre cette question à la mémoire de l'ordinateur? Les techniques varient selon les systèmes qu'on utilise. Si l'on utilise un système à vocabulaire contrôlé, il faudra d'abord chercher dans le thesaurus les termes définis et acceptés qui expriment le sujet. Si l'on utilise un système à vocabulaire libre, on pourra utiliser les termes mêmes de la question, mais en plus chercher d'autres termes connexes susceptibles d'être utilisés par les auteurs dans leurs articles. Supposons que nous utilisons dans notre exemple un système à vocabulaire libre, de modèle KWIC.

La première étape consiste à isoler les descripteurs considérés comme importants pour le repérage de la documentation. Dans l'exemple apporté plus haut, les descripteurs importants sont les suivants:

- audio-visuel

- enseignement

- universités

- collèges.

Mais il ne suffit pas de soumettre à la mémoire de l'ordinateur ces descripteurs isolés; car alors l'ordinateur chercherait les documents qui contiennent l'un ou l'autre de ces descripteurs et fournirait une foule de références qui n'auraient rien à voir avec le sujet: Exemples:

- L'audio-visuel et la publicité

- Situation de l'enseignement supérieur au Québec 
- L'administration des universités contemporaines

- La place des bibliothèques das les collèges d'enseignement général

- etc...

Aussi la seconde étape consiste à établir des liens logiques entre les descripteurs retenus. C'est ici qu'entrent en jeu les règles de construction du profil, qui reposent sur celles de la logique booléenne. Les relations logiques couramment employées dans le repérage automatique de la documentation sont les relations ET, OU et NON (intersection, réunion et exclusion de classes).

La relation ET entre deux descripteurs signifie que le titre de l'article, pour être repéré, doit contenir à la fois les deux descripteurs. La relation OU signifie que l'article doit contenir l'un ou l'autre des deux descripteurs; la relation NON signifie qu'il doit contenir le premier, mais non le second. Ainsi par exemple si on donne à l'ordinateur la relation logique suivante:

Audio-visuel

$$
\text { ET Universités }
$$$$
\text { OU }
$$

Collèges

l'ordinateur fournira tous les titres qui contiennent à la fois les termes audio-visuel et universités, de même que tous les titres qui contiennent à la fois les termes audio-visuel et collèges. La pertinence des documents fournis sera déjà beaucoup plus élevée.

On peut aussi généralement fournir à l'ordinateur des éléments de repérage autres que les termes ou concepts relatifs au contenu de la recherche. On peut lui demander par exemple de nous signaler tous les articles publiés par tel auteur; ou encore tous les documents publiés par telle association, ou encore tous les articles parus dans telle revue. A l'inverse, on peut lui demander aussi de ne pas nous fournir les articles écrits par tel auteur, etc.... II suffit d'inclure ces noms dans le profil d'intérêt et d'établir les relations logiques nécessaires.

Par exemple, notre client fictif pourrait être intéressé par tous les articles de Bernard Lachance; on n'a alors qu'à soumettre ce nom à la mémoire de l'ordinateur, à titre de descripteur isolé. II pourrait par contre se montrer non intéressé à recevoir les références aux articles de Jacques Demers, parce qu'il connaît bien tout ce qu'il écrit; ou encore non intéressé à recevoir les titres des articles qui paraissent dans la revue Journal de l'audiovisuel, parce qu'il la lit régulièrement, etc... Le profil pourrait alors s'élaborer comme suit: Première question: LACHANCE, B. (terme isolé)

Deuxième question:

Audio-visuel

NON
Collèges
Demers, J.
OU

Journal de l'audio-
visuel.

Dans ce cas, l'ordinateur fournirait:
1) tous les titres des articles écrits par Bernard Lachance;

2) tous les titres des articles contenant à la fois les termes audio-visuel et universités, de même que tous ceux contenant à la fois les termes audio-visuel et collèges; toutefois, il les rejèterait automatiquement s'ils étaient écrits par Jacques Demers ou s'ils étaient parus dans le Journal de l'audiovisuel.

On peut ainsi continuer à élaborer le profil d'intérêt, en respectant les règles prescrites, de façon à parvenir à la plus grande pertinence possible. Pour en revenir à notre exemple, on pourrait entre autres dépouiller la notion d'audio-visuel pour isoler tous les termes couvrant des techniques audiovisuelles spécifiques, comme par exemple l'enseignement télévisé, l'utilisation du film, etc.... et inclure ces nouveaux descripteurs, avec leurs relations logiques appropriées, dans le profil d'intérêt. On peut en arriver ainsi à un profil assez élaboré et complexe.

En général, les systèmes offrent aussi la possibilité de tronquer les mots. L'avantage est alors le suivant: si on inscrit dans le profil l'expression collège, l'ordinateur ne nous fournira que les références qui contiennent cette expression. Si par contre on inscrit l'expression colleg/, l'ordinateur nous fournira aussi les références qui contiennent les expressions collégial, collégiens, collèges, etc... II s'agit de tronquer les termes au bon endroit pour qu'ils gardent toute leur signification.

L'essentiel, dans l'élaboration d'un profil d'intérêt, est donc de bien comprendre l'usager, de bien connaître tous ses besoins, et ensuite de les analyser de la façon la plus exhaustive et la plus logique possible. Tels sont les principes de base de l'élaboration d'un profil; encore une fois, les élements de repérage et les techniques peuvent varier seIon les divers systèmes utilisés.

Une fois le profil construit et transmis à la mémoire de l'ordinateur, le travail n'est pas terminé pour autant. II faudra encore analyser les références reçues pour voir si elles sont vraiment pertinentes. Si on s'aperçoit qu'il y a trop de bruit, ou pas assez de pertinence, il faut alors se poser des questions...

"Searching failures are of three principal types:

1. Pure errors involving the use of inappropriate terms or defective search logic;

2. Failures due to the levels of specificity and/or exhaustivity adopted in searching strategies;

3. Recall failures due to the fact that the searcher did not cover all reasonable approaches to the retrieval of relevant literature." 19

Pour remédier à un manque de pertinence ou d'efficacité, il faudra donc introduire de nouveaux descripteurs, en éliminer certains déjà inscrits, ou encore étendre le champ de la recherche ou le limiter, introduire de nouveaux liens logiques ou assouplir les relations 19. F Wilfred LANCASTER, Information Retrieval ..., 143. 
logiques déjà établies, etc... En d'autres mots, il faut, dans tous les cas, mettre sur pied une évaluation continuelle du profil d'intérêt et de sa rentabilité, et ce, en vue d'en arriver à une efficacité idéale.

\section{CONCLUSION}

L'automatisation de la référence est donc d'un grand secours pour le bibliothécaire qui doit faire face à des demandes de plus en plus pressantes de la part des usagers, surtout au niveau de l'enseignement, des études supérieures et de la recherche. Grâce à l'automatisation de la référence, le bibliothécaire, tout comme le chercheur d'ailleurs, peut éviter les tâches accaparantes, routinières et fastidieuses, comme par exemple la compilation manuelle d'index et de répertoires. II peut aussi en arriver à une efficacité une rapidité et une pertinence supérieures à celles de la référence traditionnelle. L'ordinateur toutefois, comme nous l'avons vu, n'enlève pas au bibliothécaire ses responsabilités intellectuelles. En conclusion on peut dire ceci: l'ordinateur ne remplace pas le bibliothécaire de référence, mais il constitue pour lui un instrument de première importance qu'il devra nécessairement apprendre à utiliser s'il veut garder, dans l'avenir, son titre de spécialiste de la documentation.

\section{BIBLIOGRAPHIE}

ADKINSON, Burton W. et DUBESTER, Henry J. "The New Challenges for University and Technical Libraries", Libri, 1969, vol.19, p.265-274.

CHAUMIER, Jacques. Les techniques documentaires. Paris, Presses universitaires de France, 1971. 128p. (Que sais-je? 1419)

DENSKY, Marguerite. "Les services de référence dans les bibliothèques canadiennes", Bulletin de l'ACBLF, 1970, vol.16, p.72-84.
HEILIGER, Edward M. et HENDERSON Jr., Paul B. Library Automation: Experience, Methodology and Technology of the Library as an Information System. NewYork, McGraw-Hill, 1971. 333p.

HEILIK, J. "Information Retrieval and MARC at the National Science Library", Canadian Library Journal, 1971, p.120-123.

KATZ, William A. Introduction to Reference Work. Vol.II: Reference Services. New-York, McGraw-Hill, 1969. 254 p.

LANCASTER, F Wilfrid. Information Retrieval Systems: Characteristics, Testing and Evaluation. New-York, John Wiley, 1968. 222p.

National Research Council of Canada. National Science Library. CAN/SDI Project. Profile Design Manual. 3rd ed. Ottawa, 1972.

PRODRICK, R.G. "Automation Can Transform Reference Services", Ontario Library Review, September 1967, p. $145-150$

ROTHSTEIN, Samuel. The Development of Reference Services through Academic Traditions, Public Library Practice and Special Librarianship. Chicago, Association of College and Reference Libraries, 1955. 124p. (ACRL Monographs, no.14)

UNISIST: Abrégé de l'Etude sur la réalisation d'un système mondial d'information scientifique, effectuée par l'Organisation des Nations Unies pour l'éducation, la science et la culture et le Conseil international des unions scientifiques. Paris, UNESCO, 1971. 95p.

VAN DIJK, Marcel et VAN SLYPE, Georges. Le service de documentation face à l'explosion de l'information. Paris, Editions d'Organisation, 1969. 265p.

\section{A votre service}

Des titres dans les catégories suivantes: Philosophie, religion, théologie, sociologie, sciences politiques, éducation, langue et littérature, histoire, biographies.

Deux périodiques: Éducation et Société, revue d'animation; Relations, revue d'intérêt général.

Demandez notre catalogue

Les Éditions Bellarmin,

8100, boul. Saint-Laurent, Montréal 351 • Tél.: 387-2541 